\title{
NATIONAL SCALE MUTLI-HAZARD MODEL PLATFORM FOR EXTREME CYCLONE IMPACTS ON COASTS AND INFRASTRUCTURE
}

\author{
David Taylor, Baird Australia, dtaylor@baird.com \\ Dr Joanna Aldridge, Griffith University, joanna aldridge@outlook.com \\ Jarrod Dent, Baird Australia, jdent@baird.com \\ Jim Churchill, Baird Australia, jchurchill@baird.com
}

\begin{abstract}
Extreme event impacts on coastlines and infrastructure is often driven by a combination of processes and factors. In cyclone (hurricane) prone coastlines, wind, rain, storm surge, tide and waves can all contribute to damage and loss of infrastructure. With increasing value of coastal assets, hazard and risk management is increasingly assessing multi-hazard impacts from extreme events, and cumulative damage from multiple events within short timeframes.
\end{abstract}

A novel multi-hazard model system has been developed to assess cyclone hazards on the Australian coastline from a combination of wind, rain, tide, storm surge and wave impacts. The model system is capable of deriving return period hazard levels for single and combined hazards, and also long-duration, for example 10,000 year duration, event sets to analyze cumulative impacts. The hazard model system is comprised of high-resolution, calibrated process models addressing the various physical processes that contribute to the overall hazard.

The fundamental component of the model system is a Monte Carlo cyclone track model system covering the whole Australian cyclone region $\left(90^{\circ} \mathrm{E}\right.$ to $\left.160^{\circ} \mathrm{E}\right)$ using the modelling approach presented in Taylor et al (2011). The track model simulates cyclogenesis, cyclone track and intensity across the whole region based on data derived relationships from over 40-years of satellite era data. The track model has achieved good model validation for landfall frequency, track and intensity around the whole of Australia (Burston et al 2015 and 2017).

The track model provides inputs for derivation of cyclone wind fields using a parametric Holland (2010) model. The cyclonic wind model has been validated for offshore and 'at coast' winds at selected locations and the derived wind hazard levels from the Monte Carlo model have good agreement with 20 to 100 -year ARI wind speed estimates derived from measured data.

The cyclone wind model provides forcing to a series of national scale hydrodynamic models that are capable of modelling tides and storm surge at a resolution of $200 \mathrm{~m}$ to $300 \mathrm{~m}$ along the entire cyclone prone region of Australia. Model validation for tides, peak storm surge and water levels is excellent.

The model systems have been applied to generate a 10,000 year event set of cyclone tracks, winds, storm tide (surge + tide) and shoreline wave conditions for the entire
Australian cyclone region. Coastal inundation has been calculated with a hydro-connectivity model at highresolution $(\approx 20 \mathrm{~m})$ using LiDAR and satellite derived data. The inundation model includes a wave module that addresses additional wave generated inundation from nearshore wave setup and runup.

The resulting data set is unique in its combined scale and resolution, covering 25,000 km's of coastline, and comprising over 100,000 events. The data set has been compiled into a geospatial database and outputs are viewable in a range of GIS platforms, including GoogleEarth as presented in Figure 1.

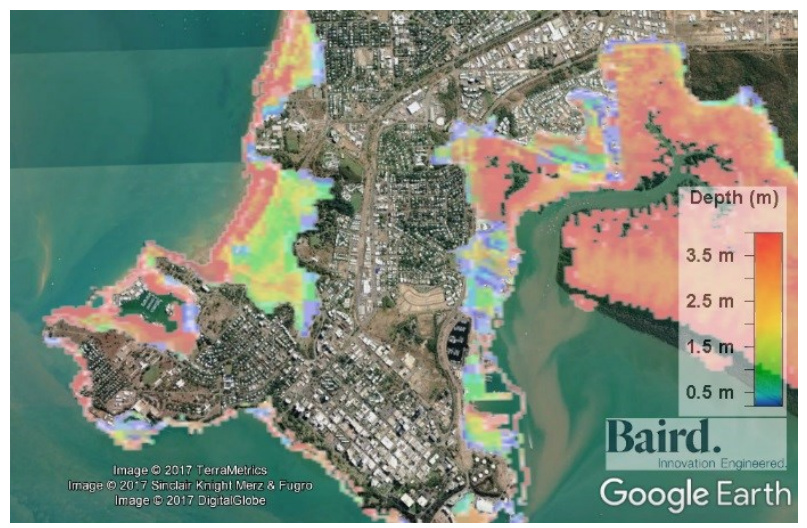

Figure 1 - Example of event inundation extent for Darwin.

The latest component of the model system has been a parametric tropical cyclone rainfall model for North Queensland (Burston, 2017) that was formed from highresolution atmospheric modelling of historical cyclone events. Further development of the model is ongoing, including high-resolution dynamic modelling of cyclone winds over urban communities.

\section{REFERENCES}

Burston, Taylor, Dent, Churchill, (2017): Australia-wide Tropical Cyclone Multi-hazard Risk Assessment. Proceedings of Coasts and Ports 2017. Cairns, June 2017.

Burston, Taylor, and Churchill J. (2015): Stochastic Tropical Cyclone Modelling in the Australian Region: An Updated Track Model. Proceedings of Coasts and Ports 2015. Auckland, September 2015.

Taylor, Branson, and Treloar, (2011): "Monte Carlo Cyclone Track Model System for the Pilbara Coast." Proceedings of Coasts and Ports 2011. Perth 2011. 